\title{
Frequency-Dependent Effects on Bladder Reflex by Saphenous Nerve Stimulation and a Possible Action Mechanism of Tibial Nerve Stimulation in Cats
}

\author{
Xing $\mathrm{Li}^{1,2}$, Xiaoping Wan ${ }^{2}$, Zhaoxia Wang ${ }^{3}$, Yanan Liang ${ }^{2}$, Zhuo Jia ${ }^{1}$, Xu Zhang ${ }^{1}$, Limin Liao ${ }^{2}$ \\ ${ }^{1}$ Department of Urology, Medical College of People's Liberation Army, Chinese People’s Liberation Army General Hospital, Beijing, China \\ ${ }^{2}$ Department of Urology, Rehabilitation School of Capital Medical University, China Rehabilitation Research Center, Beijing, China \\ ${ }^{3}$ Department of Rehabilitation Medicine, Beijing Tiantan Hospital, Capital Medical University, Beijing, China
}

Purpose: The present study determined the effects of saphenous nerve stimulation (SNS) at different stimulation frequencies on bladder reflex and explored a possible action mechanism of tibial nerve stimulation (TNS) on bladder activity in cats.

Methods: Two bipolar nerve cuff electrodes were implanted on the saphenous nerve and the contralateral tibial nerve in 13 cats, respectively. Multiple cystometrograms were obtained to determine the effects of single SNS at different frequencies and that of combined SNS and TNS on the micturition reflex by infusing normal saline.

Results: SNS at $1 \mathrm{~Hz}$ significantly reduced the bladder capacity (BC) to $59.8 \% \pm 7.7 \%$ and $59.3 \% \pm 5.8 \%$ of the control level at the intensity threshold $(\mathrm{T})$ and $2 \mathrm{~T}$, respectively $(\mathrm{P}<0.05)$, while that at $20 \mathrm{~Hz}$ significantly increased the $\mathrm{BC}$ to $130.6 \% \pm 4.2 \%$ of the control level at $6 \mathrm{~T}(\mathrm{P}<0.05)$. The TNS and SNS at $20 \mathrm{~Hz}$ did not significantly change the $\mathrm{BCs}$ at $1 \mathrm{~T}(\mathrm{P}>0.05)$, while combined stimulation at $1 \mathrm{~T}$ significantly increased the $\mathrm{BC}$ to $122.7 \% \pm 1.9 \%$ of the control level and induced an inhibitory effect which was similar to that TNS at 2T.

Conclusions: The current study revealed that SNS reduced and increased BC depending on different stimulation frequencies. The combined SNS and TNS maximized the clinical efficacy at a low intensity. Also, SNS may be a potential therapeutic mechanism of TNS.

Keywords: Urinary bladder, Overactive; Saphenous nerve; Tibial nerve stimulation; Urinary bladder, Underactive

- Fund/Grant Support: This study was funded by the China Postdoctoral Science Foundation funded project (2018M643880) and Beijing Natural Science Foundation (No. 7182188).

- Research Ethics: All applicable international, national, and/or institutional guidelines for the care and use of animals were followed. All experiments in this study were approved by the animal care and use committee at Capital Medical University (AEEI-2020-141).

- Conflict of Interest: No potential conflict of interest relevant to this article was reported.

\footnotetext{
- HIGHLIGHTS

- Saphenous nerve stimulation have different effects on bladder reflex depending on stimulus frequency.

- Also, it may be a potential therapeutic mechanism of tibial nerve stimulation.
}

Corresponding author: Xu Zhang (iD https://orcid.org/0000-0000-0000-0000 Department of Urology, Chinese People's Liberation Army General Hospital, No 28. Fuxing Road, Haidian District, Beijing 100853, China Email: xzhang@foxmail.com

Corresponding author: Limin Liao (ib https://orcid.org/0000-0000-0000-0000

Department of Urology, China Rehabilitation Research Center, No 10. Jiaomen

North Road, Fengtai District, Beijing 100068, China

Email:1mliao@263.net

Submitted: August 11, 2020 / Accepted after revision: August 23, 2020
This is an Open Access article distributed under the terms of the Crecc)
commons.org/licenses/by-nc/4.0/) which permits unrestricted non-commercial use, distribution, and reproduction in any medium, provided the original work is properly cited. 


\section{INTRODUCTION}

Sacral neuromodulation or sacral nerve stimulation is the U.S. Food and Drug Administration-approved therapy that treats 2 opposite bladder conditions: overactive bladder $(\mathrm{OAB})$ and nonobstructive urinary retention, which is a characteristic of the underactive bladder (UAB) [1-3]. However, this technique has not been applied widely because it is 2-staged invasive and complicated implantation process. Also, many patients cannot afford the high cost of the procedure. Hence, increasing attention has been focused on exploring the effects of minimally invasive peripheral nerve electrical stimulation on the bladder function.

Tibial nerve stimulation (TNS) is a third-line treatment option for $\mathrm{OAB}$ patients who are refractory to conservative therapies [4]. The stimulation amplitude is equal to or below the intensity threshold (T) inducing foot motor [5]. However, animal studies indicated that required intensities causing bladder inhibition are greater than those used clinically [6,7]. This disparity is currently under investigation. Anatomical studies showed that the posterior branch separated from the main trunk of the saphenous nerve passes through the approximate location where the needle electrode of percutaneous TNS (PTNS) or the surface electrode of transcutaneous TNS (TTNS) is placed [8,9]. The animal studies in rats and clinical investigation confirmed that saphenous nerve stimulation (SNS) at $20-\mathrm{Hz}$ frequency inhibits the bladder reflex and be used for treating OAB [10-12]. Therefore, some researchers speculated that the saphenous nerve is coactivated, and there may be a potential action mechanism during TNS therapy [13]. Thus, animal experiments are needed to test this hypothesis.

Interestingly, a recent animal study in cats showed that SNS at $1-\mathrm{Hz}$ frequency induced bladder excitation and restored the bladder capacity (BC) to normal level [14]. The present study provided a novel neuromodulation therapy for UAB that is valuable when the reflex of the bladder with a large capacity is underactive. Based on the above studies, it seems that the effects of SNS on the bladder function depend on the frequency of stimulation. Previous studies demonstrated that pudendal nerve stimulation (PNS) at $3-15 \mathrm{~Hz}$ inhibits the bladder, while stimulation at $20-50 \mathrm{~Hz}$ evokes bladder contractions $[15,16]$. Similarly, TNS at low frequency $(2 \mathrm{~Hz})$ and high frequency $(5-$ $20 \mathrm{~Hz}$ ) evokes excitatory and inhibitory bladder responses, respectively [17]. Our recent study also showed that sacral dorsal root ganglion (DRG) electrical stimulation at low frequencies
(3-7 Hz) inhibited isovolumetric rhythmic bladder contractions, while excitatory effects were observed at 2 frequency ranges: low frequencies $(0.25-1.5 \mathrm{~Hz})$ and middle frequencies $(15-30 \mathrm{~Hz})$ [18]. Irrespective of SNS at 1-Hz frequency under normal bladder reflex activity, excitatory bladder effects have yet to be investigated.

The goals of the current study were as follows: (1) to determine the effects of different SNS stimulation frequencies on bladder reflex with normal saline infusion; (2) to evaluate the effects of combined SNS and TNS stimulation on bladder activity to explore a putative TNS therapeutic mechanism.

\section{MATERIALS AND METHODS}

\section{Surgical Procedure}

The experiments were conducted in 13 randomly selected adult cats ( 9 males and 4 females, 6-12 months old, weighing 2.3-3.1 $\mathrm{kg}$ ). The animal care and use committee at Capital Medical University approved all protocols involving the use of animals in this study (AEEI-2020-141). Each animal was anesthetized with isoflurane ( $2 \%-5 \%$ in oxygen) during surgery and switched to intravenous $\alpha$-chloralose $(65 \mathrm{mg} / \mathrm{kg}$, supplemented as needed) during data acquisition. The anesthetic and fluid were administered via the left cephalic vein. The heart rate and blood oxygen level were monitored throughout the experiment. The body temperature was maintained by a heating pad. A middle abdominal incision was made to expose the bladder, and the ureters were separated (the left was ligated, and the right was externally drained). A 2-lumen catheter was introduced into the bladder via a small cut on the proximal urethra and secured by a ligature around the urethra. One lumen of the catheter was used to infuse the bladder with normal saline at the rate of $1-2 \mathrm{~mL} / \mathrm{min}$ after connecting to a pump. The other lumen was connected to a pressure transducer (MP150; BIOPAC Systems, Inc., Camino Goleta, CA, USA) to measure the bladder pressure.

The left saphenous nerve was exposed via an incision along the medial aspect slightly above the knee joint. Another incision was made on the medial side of the contralateral hindlimb above the ankle to expose the tibial nerve for stimulation to avoid the ipsilateral muscle excessive fatigue. Two custom-fabricated bipolar nerve cuff electrodes (platinum contacts) were implanted on the 2 nerves, and electrical pulses were given out by an external stimulus generator (Master-8; AMPI, Jerusalem, Israel). Subsequently, the incisions were closed after the surgery. 


\section{Stimulation Protocol}

Approximately 30 minutes after surgery, a series of acute experiments were started. BC was used to test the inhibitory or excitatory effects of the stimulation. The bladder was drained before each experiment. After the appearance of the first large amplitude ( $>30 \mathrm{~cm} \mathrm{H}_{2} \mathrm{O}$ ) and long duration ( $>20$ seconds) bladder contraction, the bladder infusion was stopped [14]. BC was defined as the volume of infusion into the bladder. Initially, after emptying the bladder, 2 or 3 cystometrograms (CMGs) were obtained without stimulation that served as the control BC.

After the BC stabilized, stimulation was applied during sequential CMGs. Uniphasic rectangular pulses (0.2-msec pulse width) were delivered to the 2 nerves. The threshold, T, was defined as the minimal intensity for inducing observable muscle twitches on the posterior thigh, hip, or toe for the saphenous nerve and toe twitches for the tibial nerve. First, the frequencydependent effects of SNS at 3 different frequencies $(1,5$, and 20 $\mathrm{Hz}$ ) were tested in a randomized order. Second, 6 CMGs were performed to evaluate the effects of combined SNS and TNS stimulation: (1) control CMG without stimulation; (2) CMG with TNS $(20 \mathrm{~Hz}, 1 \mathrm{~T})$; (3) CMG with SNS $(20 \mathrm{~Hz}, 1 \mathrm{~T})$; (4) CMG with TNS (20 Hz, 2T); (5) CMG with combined SNS and TNS stimulation $(20 \mathrm{~Hz}, 1 \mathrm{~T})$; (6) control CMG again to determine any poststimulation effect. The bladder was emptied after each CMG, pausing for a 3-4-minutes rest between successive CMGs to allow the bladder to recover.

\section{Statistical Analysis}

Prism ver. 8.0 (GraphPad Software, La Jolla, CA, USA) was used for statistical analysis. To compare the sequential CMG recordings, the BCs were normalized to the measurement of the first control CMG, and calculated as follows: normalized BCs= $\mathrm{Cs} / \mathrm{BC}$ of the first control CMG $\times 100 \%$. The amplitude of maximal bladder contractions was also measured. Repeated measurements under the same conditions in the same animal were averaged. All summary data are expressed as the mean \pm standard error. One-way analysis of variance, followed by Bonferroni multiple comparisons, was used to determine the statistical significance $(\mathrm{P}<0.05)$.

\section{RESULTS}

\section{Frequency-Dependent Effects of SNS on Bladder Reflex}

SNS at $1 \mathrm{~Hz}$ significantly reduced BC to $59.8 \% \pm 7.7 \%$ and $59.3 \% \pm 5.8 \%$ of the control level at $1 \mathrm{~T}$ and $2 \mathrm{~T}$, respectively
$(\mathrm{P}<0.05)$. However, no significant difference was detected between the 2 intensities $(\mathrm{P}>0.05)$ (Fig. 1). When examined at $5-\mathrm{Hz}$ frequency, no significant effects on the $\mathrm{BC}$ were detected in the results obtained using 1-6T intensities $(\mathrm{P}>0.05)$ (Fig. 2). SNS $(20 \mathrm{~Hz})$ at intensities of $1-4 \mathrm{~T}$ did not significantly change the BCs $(\mathrm{P}>0.05)$, while it was significantly increased to $130.6 \% \pm 4.2 \%$ of the control level at $6 \mathrm{~T}(\mathrm{P}<0.05)$ (Fig. 3). Moreover, the BCs returned to the prestimulation levels without any poststimulation effects at the 3 frequencies and SNS at 1-15 Hz did not significantly change the maximal contraction amplitude of micturitions (Figs. 1-3).

\section{Combined Bladder Inhibition of Simultaneous SNS and TNS}

TNS and SNS at $20 \mathrm{HZ}$ did not significantly change the BCs at $1 \mathrm{~T}$, respectively $(\mathrm{P}>0.05)$, while TNS at $2 \mathrm{~T}$ significantly increased the BC to $128.3 \% \pm 3.8 \%$ of the control level $(\mathrm{P}<0.05)$ (Fig. 4). When SNS was combined with TNS at 1T, it significantly increased the $\mathrm{BC}$ to $122.7 \% \pm 1.9 \%$ of the control level and induced an inhibitory effect similar to that TNS at 2T (Fig. 4). The combined stimulation also significantly increased the $\mathrm{BC}$ compared to separated TNS and SNS at $1 \mathrm{~T}(\mathrm{P}<0.05)$ (Fig. 4). There were no significantly change about the maximal contraction amplitude of micturitions during different nerve stimulation (Fig. 4).

\section{DISCUSSION}

The results of the current study revealed a frequency-dependent role of SNS as it could not only decrease the $\mathrm{BC}$ at $1 \mathrm{~Hz}$ but also inhibit the bladder activity and increase the $\mathrm{BC}$ at $20 \mathrm{~Hz}$ (Figs. $1,3)$. Previous studies suggested that the stimulating frequency is a critical parameter that mediates the modulatory effect on bladder activity. A study of electrical stimulation of pudendal nerve trunk in cats showed that PNS at $3 \mathrm{~Hz}$ inhibited the reflex bladder activity and significantly increased the $\mathrm{BC}$, while that at $20 \mathrm{~Hz}$ induced large bladder contractions [15]. Another 2 studies about selective stimulation of pudendal nerve branches in the cat also reported the frequency-dependent characteristics: inhibited detrusor activity of dorsal genital nerve $(5-10 \mathrm{~Hz})$ and sustained bladder contractions of cranial sensory $(\leq 5 \mathrm{~Hz})$, dorsal genital $(\geq 20 \mathrm{~Hz})$, and rectal perineal $(\leq 10 \mathrm{~Hz})[16,19]$. Moazzam et al. [17] performed an acute study in anesthetized cats and concluded that TNS at low frequency $(2 \mathrm{~Hz})$ and high frequency $(5-20 \mathrm{~Hz})$ could evoke excitatory and inhibitory 

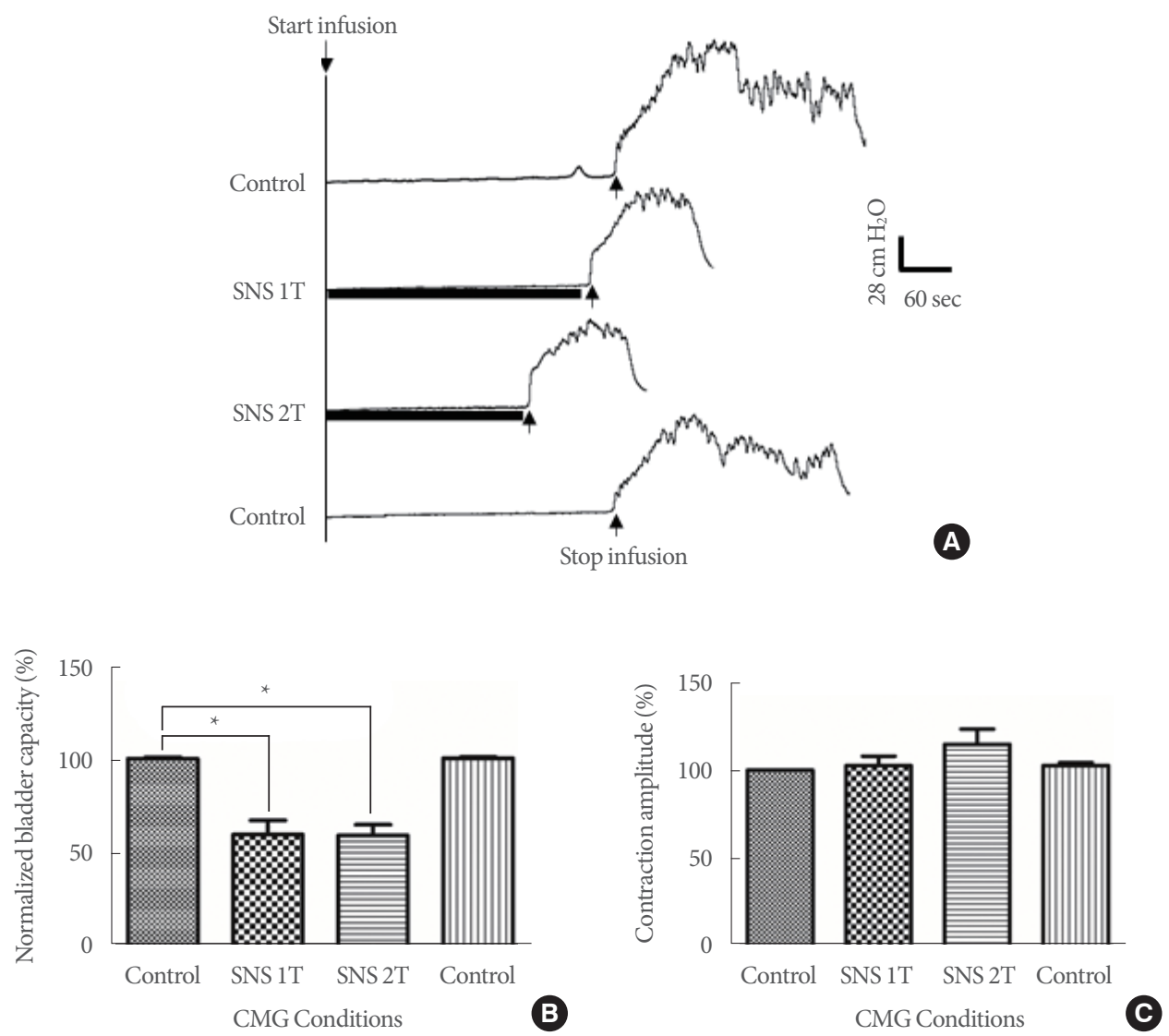

Fig. 1. Saphenous nerve stimulation (SNS) applied at $1 \mathrm{~Hz}$ significantly reduced the bladder capacity. (A) Repeated cystometrogram (CMG) traces with/without SNS. The black bar under bladder pressure traces indicates the duration of stimulation. Stimulation: 0.2msec pulse width; threshold intensity (T) for inducing muscle twitching on the toe $=0.76 \mathrm{~V}$; infusion rate: $2 \mathrm{~mL} / \mathrm{min}$. (B) Summarized bladder capacities measured during different CMGs. T $=0.18-1.18 \mathrm{~V}$; infusion rate: $1-2 \mathrm{~mL} / \mathrm{min}$. (C) Summarized results of contraction amplitudes. ${ }^{*}$ Significantly different from the control CMG.

bladder responses, respectively. Our recent study in cats also showed that TNS $(0.5-3 \mathrm{~Hz})$ induced large amplitude and sustained bladder contractions when the bladder was underactive, while in OABs, TNS inhibited the bladder overactivity at $5 \mathrm{~Hz}$ [20]. Another our recent study in cats explored the excitatory and inhibitory effects of stimulation of sacral DRG on bladder reflex in cats [18]. Herein, we found that stimulation at low frequencies (3-7 Hz on S1 or S2 DRG) significantly inhibited isovolumetric bladder contractions, while excitatory effects were observed at 2 frequency ranges: low frequencies $(0.25-1.5 \mathrm{~Hz}$ on S1 DRG and $0.25-1.25 \mathrm{~Hz}$ on S2 DRG) and middle frequencies (15-30 Hz on S1 and S2 DRGs). But we did not investigate the effects of contraction amplitude and time during the voiding phase and whether SNS at $1 \mathrm{~Hz}$ had an excitatory effect or not must be explored in the future study.

Compared to the TNS, there are only a few studies about
SNS. The study by MacDiarmid et al. [10] presented the initial experience in treating OAB patients by SNS. Electrical stimulation was applied at a frequency of $20 \mathrm{~Hz}$ for 30 minutes and repeated weekly for 12 weeks. Positive response to SNS was achieved in 14 of 16 patients (87.5\%), and no obvious adverse events were reported. Two studies investigated the acute effects of SNS in rats at different frequencies $(2,5,10,20$, and $50 \mathrm{~Hz})$ $[11,12]$. The studies indicated that SNS at $20 \mathrm{~Hz}$ evoked bladder inhibitory effects and was effective in increasing the $\mathrm{BC}$, which was in agreement with the current results. Other frequencies resulted in nonsignificant effects. Our results showed that SNS at $20 \mathrm{~Hz}$ inhibited bladder activity, while that at $5 \mathrm{~Hz}$ did not have any effect on the bladder activity even if applied at a high intensity (6T) (Figs. 2, 3). However, TNS at both $5 \mathrm{~Hz}$ and 20 $\mathrm{Hz}$ could inhibit the bladder activity at lower intensity (2T-4T) as compared to SNS $(20 \mathrm{~Hz}, 6 \mathrm{~T})$, indicating a difference be- 

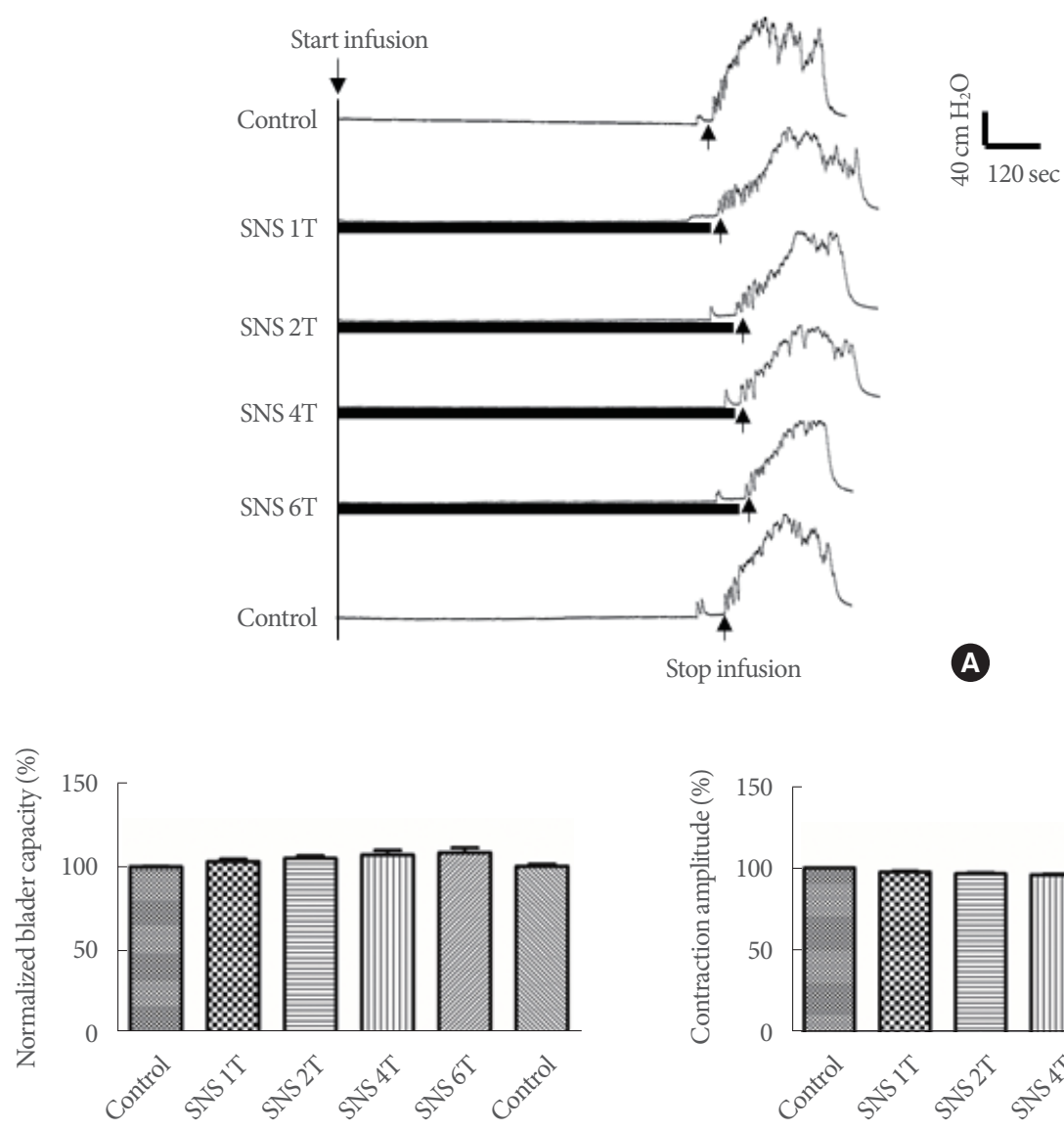

CMG Conditions

B

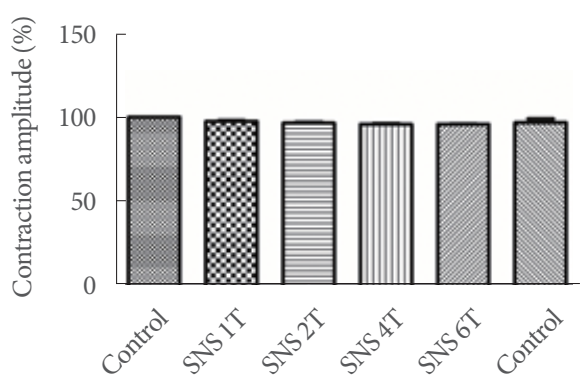

CMG Conditions

(

Fig. 2. Saphenous nerve stimulation (SNS) applied at $5 \mathrm{~Hz}$ had no significant effects on bladder capacity. (A) Repeated cystometrogram (CMG) traces with/without SNS. The black bar under bladder pressure traces indicates the duration of stimulation. Stimulation: 0.2-msec pulse width; threshold intensity (T) for inducing muscle twitching on the back of the thigh $=1.48 \mathrm{~V}$; infusion rate: $2 \mathrm{~mL} / \mathrm{min}$. (B) Summarized bladder capacities measured during different CMGs. T=0.2-2.3 V; infusion rate: $1-2 \mathrm{~mL} / \mathrm{min}$. (C) Summarized results of contraction amplitudes.

tween the 2 types of peripheral nerve neuromodulations. Due to the superficial location of the saphenous nerve, SNS may also be achieved with a chronically implanted device or by a transcutaneous modality similar to TNS used in clinical practice $[21,22]$. The inhibited effects of SNS need to be validated in further prospective, randomized, controlled studies. A recent study establishing the animal model of UAB in cats showed that SNS at $1-\mathrm{Hz}$ frequency induced bladder excitation and restored the $\mathrm{BC}$ to normal level when the bladder reflex is underactive with a large $\mathrm{BC}$ [14]. The current study investigated the effects of SNS at $1 \mathrm{~Hz}$ frequency under normal bladder reflex activity (Fig. 1). It could significantly reduce the $\mathrm{BC}$ as compared to the control level. Our recent study also established an animal model of UAB in cats by PNS [23]. Additional studies are essential to verify whether SNS at $1 \mathrm{~Hz}$ under a PNS-induced underactive state is effective. Interestingly, a similarity was noted between TNS and SNS as both could evoke excitatory effect at a low-frequency stimulation (TNS at $2 \mathrm{~Hz}$ and SNS at $1 \mathrm{~Hz}$ ) $[14,20]$.

In clinical practice, TNS is recommended as a third-line therapy for treating $\mathrm{OAB}$ [24]. The stimulation intensity is confirmed by the appearance of toe fanning or sensations radiated along the foot. However, most patients have been reported to experience painful sensations at the motor $\mathrm{T}$ [25]. Hence, it is set at one level equal to or below the intensity $\mathrm{T}$ [5]. On the contrary, significant inhibitory effects are evoked at $2 \mathrm{~T}-4 \mathrm{~T}$ in anesthetized cats and 3T-6T in anesthetized rats $[6,7,17,26]$. The saphenous trunk separates into major branches, which innervate the anterior and posterior regions of the lower leg in 

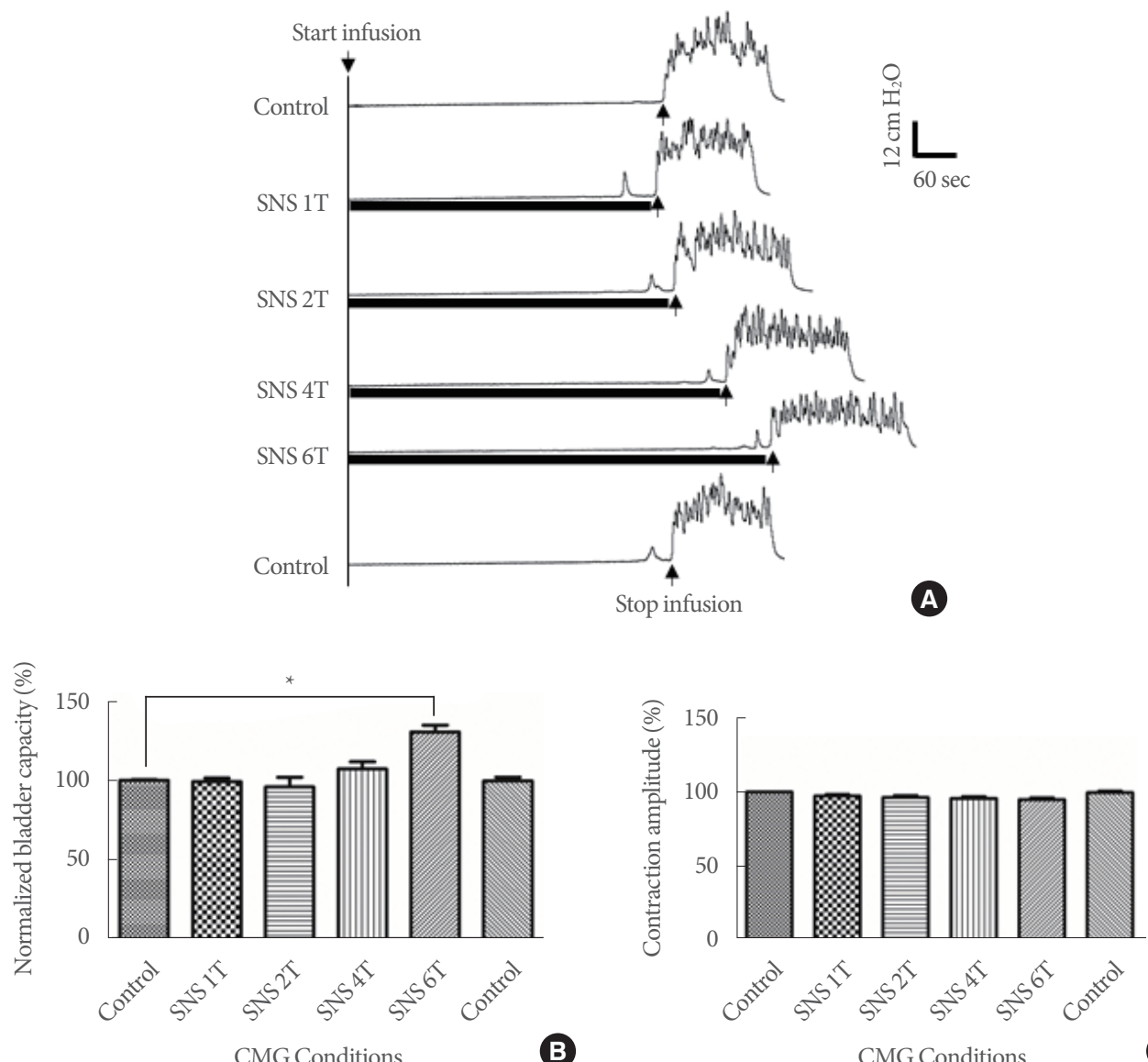

CMG Conditions

B

CMG Conditions

C

Fig. 3. Saphenous nerve stimulation (SNS) applied at $20 \mathrm{~Hz}$ significantly increased the bladder capacity and reduced contraction amplitude. (A) Repeated cystometrogram (CMG) traces with/without SNS. The black bar under bladder pressure traces indicates the duration of stimulation. Stimulation: $0.2-\mathrm{msec}$ pulse width; threshold intensity (T) for inducing muscle twitching on the toe $=0.18 \mathrm{~V}$; infusion rate: $2 \mathrm{~mL} / \mathrm{min}$. (B) Summarized bladder capacities measured during different CMGs. T=0.18-1.48 V; infusion rate: 1-2 $\mathrm{mL} / \mathrm{min}$. (C) Summarized results of contraction amplitudes. ${ }^{*}$ Significantly different from the control CMG.

humans [8]. The posterior branch appears to overlap the region where the stimulation electrodes are applied during PTNS/ TTNS therapy $[8,9,12]$. In addition, the preclinical and clinical studies demonstrated that SNS at 20-Hz frequency inhibits the bladder reflex and is used for treating OAB [10-12]. Therefore, it is hypothesized that the saphenous nerve is coactivated during TNS treatment and is a potential therapeutic mechanism of TNS therapy [10-13]. However, to the best of our knowledge, the coactivated mechanism has not yet been investigated in animal experiments. The current results proved for the first time that SNS was involved in the process of TNS. TNS or SNS at 1T alone did not inhibit the bladder reflex. However, the combined stimulation at low intensity $(1 \mathrm{~T})$ significantly increased the BC and suppressed the bladder activity, which did not differ significantly from the stimulation effects by TNS at $2 \mathrm{~T}$ (Fig. 4). Our initial results indicated that (1) SNS played a role in the inhibition of the bladder activity during TNS, rendering it to be a potential underlying therapeutic mechanism; (2) SNS in combination with TNS maximized the clinical efficacy at a low intensity that could be a novel treatment modality for OAB. Single SNS at $20 \mathrm{~Hz}$ inhibited the bladder activity at a high intensity (6T); however, only a low intensity (1T) was required during the combined stimulation. Notably, the small stimulus effect induced by SNS is indeed the "barrier" that TNS must pass through from $1 \mathrm{~T}$ to $2 \mathrm{~T}$.

Nevertheless, the present study has some limitations. First, we only investigated the acute effects of SNS and combined neurostimulation in the current study, while TNS and SNS are delivered chronically in patient stimulation $[10,25]$. Thus, the effects of long-term therapy warrant further studies. Second, we 


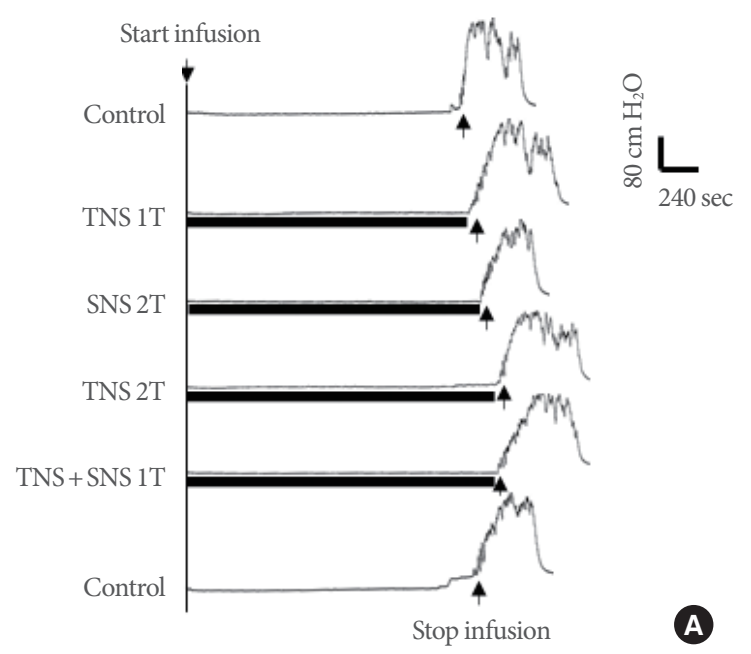

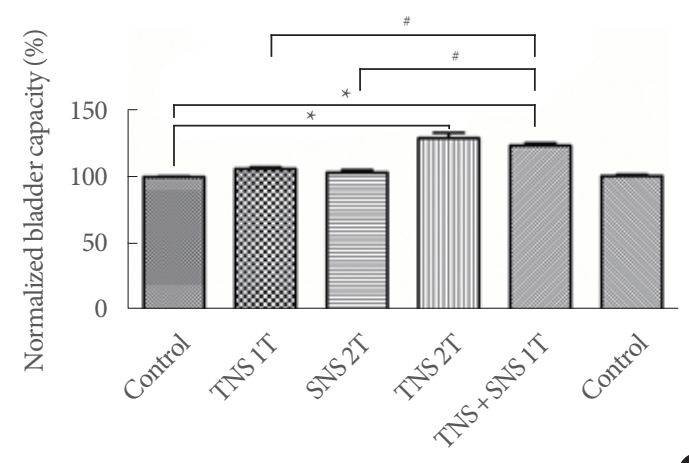

CMG Conditions

B

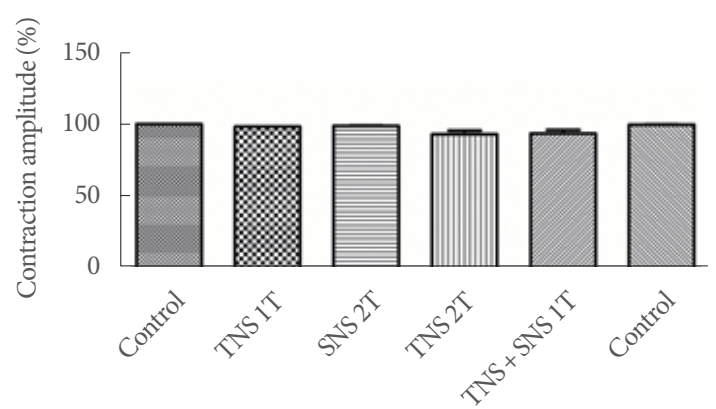

CMG Conditions

Fig. 4. Combined saphenous nerve stimulation (SNS) and tibial nerve stimulation (TNS) applied at $20 \mathrm{~Hz}$ increased the bladder capacity and reduced contraction amplitude. (A) Repeated cystometrogram (CMG) traces with/without TNS and/or SNS. The black bar under bladder pressure traces indicates the duration of stimulation. Stimulation: 0.2-msec pulse width; threshold intensity (T) for inducing muscle twitching on the toe $=0.32 \mathrm{~V}$; infusion rate: $2 \mathrm{~mL} / \mathrm{min}$. (B) Summarized bladder capacities measured during different CMGs. T=0.21-2.0 V; infusion rate: $1-2 \mathrm{~mL} / \mathrm{min}$. (C) Summarized results of contraction amplitudes. ${ }^{*}$ Significantly different from the control CMG. " Significantly different from the separate stimulation CMG.

established a study for the novel neuromodulation and provided the initial results of SNS at 3 different frequencies. A range of different frequencies from low to high remains to be explored in future studies. It is also necessary to determine the stimulation frequency that is safe for long-term usage. Third, bladder reflex contractions in the current study were evoked by normal saline infusion, which primarily activated the nonnociceptive bladder afferent fibers under physiological conditions. However, under pathological conditions, bladder irritation by acetic acid infusion activates nociceptive afferent fibers [27]. Although similar results were observed in the studies involving cats infused with saline and acetic acid [28], additional studies are imperative to confirm the effects of SNS in OAB cats. In the last, we stopped the nerve stimulation immediately before bladder contraction and did not investigate the effects of SNS during the voiding phase. whether SNS at $1 \mathrm{~Hz}$ had an excitatory effect or not must be explored in the future study.

In summary, the current study indicated that SNS reduced and increased BC depending on different stimulation frequencies. The combination of SNS and TNS maximized the clinical efficacy at a low intensity, and SNS may be a potential therapeutic mechanism of TNS. Therefore, additional preclinical and clinical studies would provide an in-depth insight into the benefits of SNS at different stimulation frequencies on long-term therapy in patients. 


\section{REFERENCES}

1. Abrams P, Cardozo L, Fall M, Griffiths D, Rosier P, Ulmsten U, et al. The standardisation of terminology of lower urinary tract function: report from the Standardisation Sub-committee of the International Continence Society. Neurourol Urodyn 2002;21:167-78.

2. Smith PP, Birder LA, Abrams P, Wein AJ, Chapple CR. Detrusor underactivity and the underactive bladder: symptoms, function, cause-what do we mean? ICI-RS think tank 2014. Neurourol Urodyn 2016;35:312-7.

3. van Kerrebroeck PE, van Voskuilen AC, Heesakkers JP, Lycklama á Nijholt AA, Siegel S, Jonas U, et al. Results of sacral neuromodulation therapy for urinary voiding dysfunction: outcomes of a prospective, worldwide clinical study. J Urol 2007;178:2029-34.

4. MacDiarmid SA, Peters KM, Shobeiri SA, Wooldridge LS, Rovner ES, Leong FC, et al. Long-term durability of percutaneous tibial nerve stimulation for the treatment of overactive bladder. J Urol 2010;183:234-40.

5. Marchal C, Herrera B, Antuña F, Saez F, Perez J, Castillo E, et al. Percutaneous tibial nerve stimulation in treatment of overactive bladder: when should retreatment be started? Urology 2011;78: 1046-50.

6. Kovacevic M, Yoo PB. Reflex neuromodulation of bladder function elicited by posterior tibial nerve stimulation in anesthetized rats. Am J Physiol Renal Physiol 2015;308:F320-9.

7. Tai C, Shen B, Chen M, Wang J, Roppolo JR, de Groat WC. Prolonged poststimulation inhibition of bladder activity induced by tibial nerve stimulation in cats. Am J Physiol Renal Physiol 2011; 300:F385-92.

8. Wilmot VV, Evans DJ. Categorizing the distribution of the saphenous nerve in relation to the great saphenous vein. Clin Anat 2013; 26:531-6.

9. Mercer D, Morrell NT, Fitzpatrick J, Silva S, Child Z, Miller R, et al. The course of the distal saphenous nerve: a cadaveric investigation and clinical implications. Iowa Orthop J 2011;31:231-5.

10. MacDiarmid SA, John MS, Yoo PB. A pilot feasibility study of treating overactive bladder patients with percutaneous saphenous nerve stimulation. Neurourol Urodyn 2018;37:1815-20.

11. Moazzam Z, Yoo PB. Electrical stimulation of the saphenous nerve in anesthetized rats: a novel therapeutic approach to treating overactive bladder. Conf Proc IEEE Eng Med Biol Soc 2016;2016:31258.

12. Moazzam Z, Yoo PB. Frequency-dependent inhibition of bladder function by saphenous nerve stimulation in anesthetized rats. Neurourol Urodyn 2018;37:592-9.
13. Elder CW, Yoo PB. Co-activation of saphenous nerve fibers: a potential therapeutic mechanism of percutaneous tibial nerve stimulation? Conf Proc IEEE Eng Med Biol Soc 2016;2016:3129-32.

14. Li S, Li X, Theisen K, Browning J, Shen B, Wang J, et al. Saphenous nerve stimulation normalizes bladder underactivity induced by tibial nerve stimulation in cats. Am J Physiol Renal Physiol 2018; 315:F247-53.

15. Tai C, Wang J, Wang X, de Groat WC, Roppolo JR. Bladder inhibition or voiding induced by pudendal nerve stimulation in chronic spinal cord injured cats. Neurourol Urodyn 2007;26:570-7.

16. Yoo PB, Woock JP, Grill WM. Bladder activation by selective stimulation of pudendal nerve afferents in the cat. Exp Neurol 2008; 212:218-25

17. Moazzam Z, Duke AR, Yoo PB. Inhibition and excitation of bladder function by tibial nerve stimulation using a wirelessly powered implant: an acute study in anesthetized cats. J Urol 2016;196:92633.

18. Wang Z, Deng H, Liao L, Lu T, Li X. Excitatory and inhibitory effects of stimulation of sacral dorsal root ganglion on bladder reflex in cats. Int Urol Nephrol 2018;50:2179-86.

19. Woock JP, Yoo PB, Grill WM. Activation and inhibition of the micturition reflex by penile afferents in the cat. Am J Physiol Regul Integr Comp Physiol 2008;294:R1880-9.

20. Theisen K, Browning J, Li X, Li S, Shen B, Wang J, et al. Frequency dependent tibial neuromodulation of bladder underactivity and overactivity in cats. Neuromodulation 2018;21:700-6.

21. van Breda HMK, Martens FMJ, Tromp J, Heesakkers JPFA. A new implanted posterior tibial nerve stimulator for the treatment of overactive bladder syndrome: 3-month results of a novel therapy at a single center. J Urol 2017;198:205-10.

22. Janssen DA, Farag F, Heesakkers JP. Urgent-SQ implant in treatment of overactive bladder syndrome: 9-year follow-up study. Neurourol Urodyn 2013;32:472-5.

23. Li X, Uy J, Yu M, Li S, Theisen K, Browning J, et al. Sacral neuromodulation blocks pudendal inhibition of reflex bladder activity in cats: insight into the efficacy of sacral neuromodulation in Fowler's syndrome. Am J Physiol Regul Integr Comp Physiol 2018;314:R3442.

24. Lightner DJ, Gomelsky A, Souter L, Vasavada SP. Diagnosis and treatment of overactive bladder (non-neurogenic) in adults: AUA/ SUFU guideline amendment 2019. J Urol 2019;202:558-63.

25. van der Pal F, van Balken MR, Heesakkers JP, Debruyne FM, Bemelmans BL. Percutaneous tibial nerve stimulation in the treatment of refractory overactive bladder syndrome: is maintenance treatment necessary? BJU Int 2006;97:547-50. 
1 Li, et al. • SNS at Different Frequencies and a Action Mechanism of TNS

26. Su X, Nickles A, Nelson DE. Comparison of neural targets for neuromodulation of bladder micturition reflex in the rat. Am J Physiol Renal Physiol 2012;303:F1196-206.

27. Fowler CJ, Griffiths D, de Groat WC. The neural control of mictu- rition. Nat Rev Neurosci 2008;9:453-66.

28. Wang Z, Liao L, Deng H, Li X, Chen G. The inhibitory effect of sacral dorsal root ganglion stimulation on nociceptive and nonnociceptive bladder reflexes in cats. World J Urol 2018;36:829-36. 